\title{
"Proyecto Sobre Ruedas": Importancia De La Prevencion De Accidentes De Trafico En La Poblacion Juvenil Andaluza
}

Luis Tallon-Aguilar ${ }^{1}$, Carmen Echevarria-Ruiz de Vargas² ${ }^{2}$ Felipe Pareja-Ciuró ${ }^{3}$, Pablo Gordillo-Fernández ${ }^{4}$, Alejandro SanchezArteaga $^{5}$, Jose Antonio Exposito-Tirado ${ }^{6}$, Jose Tinoco-Gonzalez ${ }^{7}$, Virginia Duran Muñoz-Cruzado ${ }^{8}$, Javier Padillo-Ruiz ${ }^{9}$

\begin{abstract}
Abstrato
Antecedentes: La prevención es una de las principales armas terapéuticas del trauma. Dentro del Plan Andaluz de Accidentabilidad existe una línea de trabajo específica dedicada a esta. Su medida estrella es el "Proyecto Sobre Ruedas" dirigido a adolescentes, principal grupo de riesgo: jóvenes entre 15 y 24 años, de género masculino.

Nuestro objetivo es realizar un análisis descriptivo y de resultados de esta acción preventiva desde su implantación hasta nuestros días.

Diseño del estudio: Se ha realizado un análisis del proyecto desde su puesta en marcha en el año 2009 analizando el número de eventos desarrollados, el número de habitantes a los que ha llegado y los resultados que conlleva en cuanto a reducción de accidentes y tipo de lesiones (fallecidos, heridos leves, heridos graves).

Resultados: Durante este periodo (2009-2018) se han realizado más de 150 eventos con un impacto poblacional superior a las 600,000 personas. El "Road Show" ha alcanzado a más de 100,000 adolescentes.

Casi todos los eventos van enfocados a la prevención de los accidentes de tráfico, principal mecanismo de lesión en este grupo poblacional. Realizando un análisis comparativo entre los datos anteriores al plan y posteriores, el número de fallecidos ha descendido un $53 \%$, los heridos graves un $60 \%$ y los leves un $16 \%$. En este grupo poblacional, la tasa de mortalidad desciende un $66 \%$, siendo la mayor de todos los grupos analizados.

Conclusiones: La prevención juega un papel primordial, siendo necesario una especial atención a los grupos poblacionales de riesgo y una adaptación a sus peculiaridades.

Aunque hasta ahora, se ha demostrado la eficacia de los mismos debemos insistir en este aspecto, así como en otros (formación, asistencia, investigación, gestión), con el objetivo de minimizar las lesiones secundarias a accidentes.
\end{abstract}

Palabras clave: Adolescentes, Educación, Trauma, Prevención.

Panamerican Journal of Trauma, Critical Care \& Emergency Surgery (2019): 10.5005/jp-journals-10030-1232

\section{Abstract}

Background: Prevention is one of the main therapeutic weapons in trauma. Within the Andalusian Accident Plan, there is a specific line of work dedicated to it. Its star measure is the "Project on Wheels" aimed at adolescents, the main risk group: young people between 15 years and 24 years of age, male gender.

Our objective is to carry out a descriptive and results analysis of this preventive action from its implementation to the present day.

Study design: An analysis of the project has been carried out since its implementation in 2009, analyzing the number of events developed, population it has reached, and the results that it entails in terms of accident reduction and type of injuries (deceased, minor injuries, and serious injuries).

Results: During this period (2009-2018), there have been more than 150 events with a population impact of more than 600,000 people. The "Road Show" has reached more than 100,000 teenagers.

Almost all events are focused on the prevention of traffic accidents, the main mechanism of injury in this population group.

Performing a comparative analysis between the data prior to the plan and later, the number of deaths has fallen by $53 \%$, the seriously injured by $60 \%$, and the mild by $16 \%$. In this population group, the mortality rate drops by $66 \%$, being the highest of all the groups analyzed.

Conclusion: Prevention plays a key role, being necessary a special attention to the population groups at risk and an adaptation to their peculiarities. Although up to now, the effectiveness of these has been demonstrated, we must insist on this aspect, as well as others (training, assistance, research, and management), with the aim of minimizing injuries secondary to accidents.

Keywords: Adolescents, Education, Prevention, Trauma.

\section{INTRODUCCION}

El trauma puede definirse como una herida o lesión caracterizada por una alteración estructural o un imbalance fisiológico resultante de la exposición aguda a alguna forma de energía mecánica, térmica, eléctrica, o química, o resultante de la ausencia de calor y oxígeno. ${ }^{1}$

El trauma, en sus diferentes tipos de presentación, constituye uno de los problemas más graves de salud pública en nuestro medio ya que es la primera causa de mortalidad en jóvenes,

\author{
1,3,5,7-9 Department of Surgery, Hospital Virgen del Rocío, Seville, \\ Andalucía, Spain \\ 2,6 Department of Rehabilitación, Hospital Virgen del Rocío, Seville, \\ Seville, Spain \\ ${ }^{4}$ Department of Consejeria de Salud, Plan Andaluz de Accidentabilidad, \\ Sevilla, Sevilla, Spain
}

Corresponding Author: Luis Tallon-Aguilar, Department of Surgery, Hospital Virgen del Rocío, Seville, Andalucía, Spain, Phone: +34653393951, e-mail: Itallona@hotmail.com

(0) The Author(s). 2019 Open Access This article is distributed under the terms of the Creative Commons Attribution 4.0 International License (https://creativecommons. org/licenses/by-nc/4.0/), which permits unrestricted use, distribution, and non-commercial reproduction in any medium, provided you give appropriate credit to the original author(s) and the source, provide a link to the Creative Commons license, and indicate if changes were made. The Creative Commons Public Domain Dedication waiver (http://creativecommons.org/publicdomain/zero/1.0/) applies to the data made available in this article, unless otherwise stated. 
How to cite this article: Tallon-Aguilar L, Echevarria-Ruiz de Vargas $C$, et al. "Proyecto Sobre Ruedas": Importancia De La Prevencion De Accidentes De Trafico En La Poblacion Juvenil Andaluza. Panam J Trauma Crit Care Emerg Surg 2019;8(1):19-23.

Source of support: Nil

Conflict of interest: None

y provoca un elevado coste en términos de sufrimiento humano y socioeconómico, así como una importante demanda de servicios. ${ }^{2}$

El análisis de los patrones observados en el trauma nos permite identificar los factores causales del mismo y desarrollar programas de prevención y control dirigidos principalmente a los grupos de mayor riesgo. ${ }^{3}$

En Andalucía, Comunidad Autónoma que engloba el sur de España con una población cercana a los 8.500 .000 habitantes y un área de $87.597 \mathrm{~km}^{2}$ por lo que representa la región más poblada del país y la segunda más extensa, ${ }^{4}$ se diseñó en el año 2007 el Primer Plan Integral de Atención a la Accidentabilidad, el cual nace del deseo y la necesidad de ofrecer una respuesta conjunta y organizada a este grave problema en nuestra región. Se centra en tres líneas de trabajo simultáneas, que se corresponden con las distintas fases que rodean a un accidente, (antes, durante y después del mismo). La primera línea está dirigida, a través de acciones preventivas intersectoriales, a evitar que el accidente se produzca; para cuando el accidente no ha podido ser evitado, desarrolla una segunda línea destinada a la prestación de la mejor asistencia sanitaria posible, potenciando la coordinación de los distintos servicios y dispositivos implicados; la tercera es la que plantea, tras el alta hospitalaria, las actuaciones necesarias que garanticen la continuidad asistencial en el domicilio en función de las secuelas sufridas por el ciudadano. ${ }^{2}$

Centrándonos en la primera línea de prevención de la accidentabilidad, una de las medidas estrella es el "Proyecto Sobre Ruedas" que va dirigido a los adolescentes, principal grupo de riesgo poblacional (jóvenes entre 15 y 24 años, principalmente de género masculino) con el objeto de prevenir los accidentes de tráfico y sus lesiones asociadas.

El objetivo del presente trabajo es realizar un análisis descriptivo y de resultados de esta acción preventiva concreta, y en su globalidad de todo el plan, desde su implantación hasta nuestros días.

\section{Metodos de Investigacion}

Se ha realizado un análisis descriptivo del proyecto desde su implantación (el plan de accidentabilidad se diseñó en 2007 aunque el proyecto sobre ruedas no se puso en marcha hasta el año 2009) y un análisis de resultados de la morbi-mortalidad regional asociada a los accidentes de tráfico desde esta fecha. Hemos analizado el número de eventos desarrollados dentro del programa de prevención, el número de habitantes que ha tenido una participación activa en el mismo y la mejora en los resultados de morbi-mortalidad asociada en cuanto a la reducción en el número de accidentes y el tipo de lesiones producidas en los mismos (fallecidos, heridos leves, heridos graves).

Se ha definido como fallecido como aquel individuo que pierde la vida durante el accidente o como consecuencia de las lesiones producidas en el mismo; herido grave como aquel que precisa de ingreso hospitalario; y herido leve, como aquel que precisa de asistencia sanitaria de cualquier índole sin necesidad de ser ingresado en un centro hospitalario.
El proyecto sobre ruedas está compuesto por una serie de eventos destinados a la población adolescente con el objeto de concienciar sobre esta problemática y promover una serie de medidas preventivas sobre los accidentes de tráfico, como pueden ser el uso obligatorio del cinturón de seguridad, el casco o evitar el consumo de alcohol. Dentro del proyecto se desarrollan diversas actividades o campañas como las siguientes:

- Red forma joven: Es una estrategia de salud dirigida a promover entornos y conductas saludables entre la gente joven de Andalucía. Basado en la acción intersectorial, en su desarrollo participan varias Consejerías de la Junta de Andalucía así como otras instituciones y agentes sociales como ayuntamientos, asociaciones de madres y padres. La estrategia consiste en acercar las actividades de promoción de la salud y de prevención de los riesgos asociados a la salud, a los entornos donde conviven jóvenes y adolescentes y otorgarles, además, un papel activo y central en dichas actividades. Pretende capacitar a chicos y chicas para que elijan las opciones más saludables en cuanto a los estilos de vida: alimentación, actividad física, sexualidad, etc., y en cuanto a su bienestar psicológico y social, así como en la prevención de la accidentabilidad, el tabaquismo y otras adicciones. Se desarrolla en los Puntos Forma Joven, ubicados en los diversos espacios frecuentados por la población adolescente y juvenil como son institutos de enseñanza secundaria, escuelas taller, universidades, espacios de ocio, comunitarios, etc.

- Campaña "te puede pasar a ti": Busca sensibilizar a los jóvenes sobre los accidentes de tráfico y los factores de riesgo implicados. Se enmarca en el proyecto Sobre ruedas, y cuenta con la colaboración de la Asociación Española para el Estudio de la Lesión Medular Espinal y de la International Road Safety Academy, siendo sus objetivos fundamentales reducir el número de accidentes de tráfico y sus consecuencias en la población juvenil de la comunidad andaluza; sensibilizar desde el espacio socioeducativo a los jóvenes sobre la evitabilidad de los siniestros y promover cambios de actitud ante conductas de riesgo, así como contribuir a una movilidad segura, responsable y sostenible. Las acciones son llevadas a cabo por un lesionado medular en accidente de tráfico (Asociación Aesleme) y un profesional de emergencias sanitarias (061). La ejecución de esta campaña se realiza en fechas anteriores a la realización del Road Show teniendo en cuenta para su ejecución la planificación y coordinación con el resto de actividades del centro educativo.

- Road show: Se basa en una original representación multimedia en el escenario, en la que se combinan una serie de testimonios de personas que intervienen en un siniestro de circulación, desde miembros de los servicios de emergencias hasta las víctimas. Los testimonios son los de todos aquellos que, de un modo u otro, intervienen en un accidente de tráfico: desde miembros de los diferentes servicios de emergencia hasta las propias víctimas y sus familiares, y se combinan con sonidos envolventes e imágenes impactantes. Las intervenciones de todos ellos se van intercalando con videos en los que se muestran imágenes de accidentes simulados pero de un gran realismo que consigue impactar en el espectador. Todo ello se ve complementado con una puesta en escena propicia para meter en ambiente al joven: a través de una iluminación especial y una música adecuada se recrea el entorno de una discoteca, para conseguir que antes del espectáculo el joven se encuentre "en su ambiente" y de esa manera se predisponga en mayor medida a aceptar cuanto va a ver y a escuchar (Figs 1 y 2 ). 


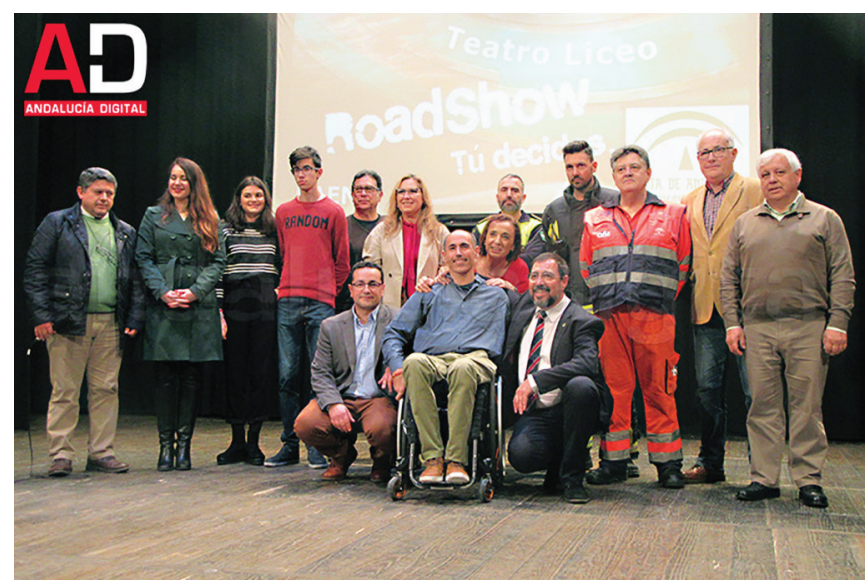

Fig. 1: Participantes de un evento road show

\section{Resultados}

El proyecto sobre ruedas durante este periodo (2009-2018) ha realizado más de 150 eventos con un impacto poblacional superior a las 600,000 personas. Dentro de éste, existen distintos módulos como el "forma joven", la Campaña "te puede pasar" (impacto de 400,000 jóvenes) o el "Road Show", evento estrella de este proyecto, que ha alcanzado a casi 100,000 adolescentes en 132 eventos.

Casi todos los eventos van enfocados a la prevención de los accidentes de tráfico ya que es el principal mecanismo de lesión en este grupo poblacional.

Si realizamos un análisis comparativo entre los datos anteriores al plan y los posteriores al mismo, observamos que el número de fallecidos global en este período en nuestra comunidad autónoma ha descendido un 53\%, los heridos graves casi un $60 \%$ mientras que los heridos leves han aumentado un 16\% (Tabla 1),

Al realizar un análisis por grupo de edad de fallecidos por accidente de tráfico en nuestra comunidad, observamos que ha habido un descenso generalizado en todos ellos salvo en el de mayor edad (superior a los 65 años). Es precisamente en este grupo poblacional de riesgo (adolescentes entre los 15 y los 24 años) donde la tasa de mortalidad desciende de forma más significativa hasta un
$66 \%$, siendo la mayor de todos los grupos por edades analizados junto al grupo entre los 25 y los 34 años (Tabla 2).

En cuanto al análisis por sexo, observamos que la tasa de mortalidad y la de accidentes sufridos es considerablemente mayor en el género masculino a nivel de todas los grupos de edad, sin existir datos completos en cuanto al descenso producido tras la puesta en marcha de los programas de prevención.

\section{Discusion}

El Plan Integral de Atención a la Accidentabilidad 2007 to 2012, se diseñó con el objetivo de contribuir a la disminución de la morbimortalidad por accidentes en la población andaluza, especialmente en la infancia (accidentes domésticos) y en los jóvenes (accidentes de tráfico), potenciando la atención e integración funcional de las personas afectadas. Se excluyen de este plan los accidentes laborales por exigir un abordaje muy específico. ${ }^{2}$

Los accidentes constituyen la primera causa de mortalidad en las edades jóvenes $y$, por tanto, de años potenciales de vida perdidos. Afectan cada año a más del $10 \%$ de la población española. Casi un tercio de ellos se producen en el hogar, seguidos de aquellos originados en la calle, correspondiendo a partes iguales los accidentes de tráfico y los debidos a otras causas. Más del $80 \%$ de los accidentes requieren algún tipo de atención sanitaria. ${ }^{2}$

La prevención juega un papel primordial en el trauma, siendo necesarias una especial atención a los grupos poblacionales de mayor riesgo y una adaptación de los programas de prevención a sus peculiaridades propias. ${ }^{3}$

Un modelo usado habitualmente en la investigación de causas de enfermedades es el conocido como el "Triángulo Epidemiológico", el cual consta de tres componentes: el huésped, el agente, y el medio ambiente. Un concepto clave es que cada componente debe ser estudiado para diseñar estrategias específicas de prevención. El huésped es la persona en riesgo para sufrir la lesión, el agente es la entidad que origina la misma (en trauma siempre es la energía aunque con diferentes mecanismos de lesión por los que es transmitida) y el medio ambiente es el escenario en el que ocurre la interacción entre el huésped y el agente. ${ }^{5}$

Las estrategias de prevención han sido recientemente categorizadas usando el concepto de las cuatro " $E$ " en relación

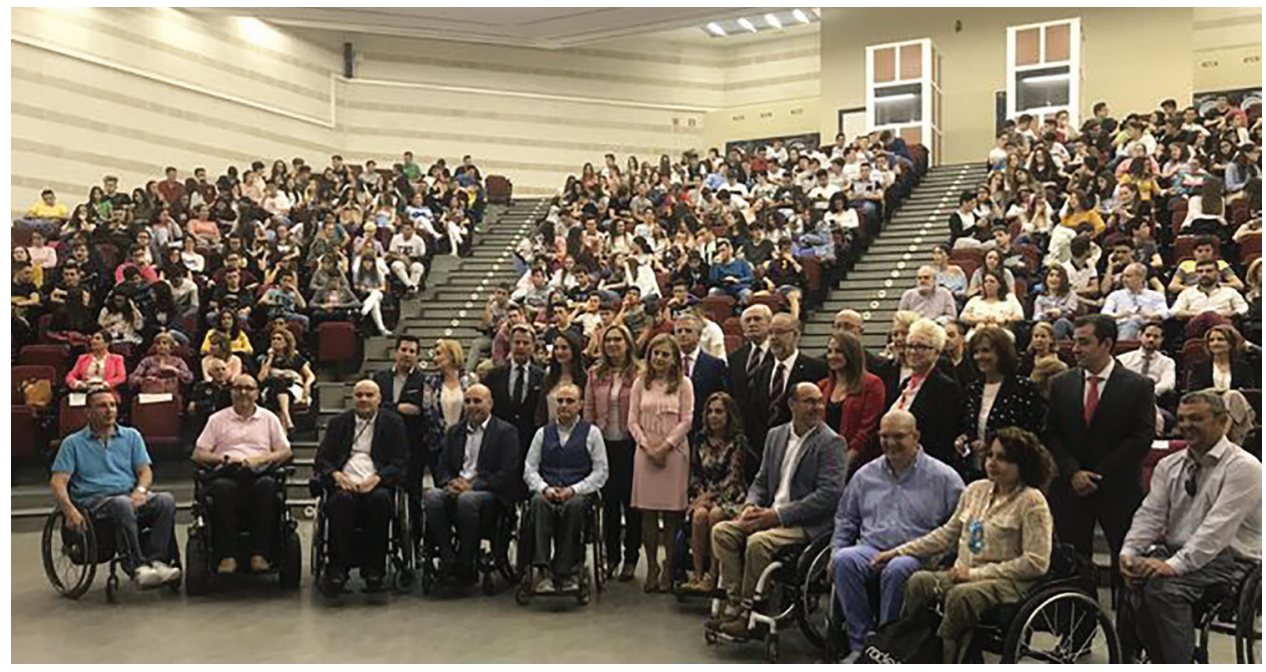

Fig. 2: Participantes y público de evento road show 
Table 1: Evolución de fallecidos, heridos graves y heridos leves por accidente de tráfico en la Comunidad Autónoma de Andalucía. Periodo 2007 to 2016 (Fuente: DGT_Dirección General de Tráfico-Ministerio del Interior. Gobierno de España. Año 2016) ${ }^{8}$

\begin{tabular}{lrrrrrrrrrrr}
\hline & \multicolumn{1}{c}{2007} & \multicolumn{1}{c}{2008} & \multicolumn{1}{c}{2009} & \multicolumn{1}{c}{2010} & \multicolumn{1}{c}{2011} & 2012 & 2013 & 2014 & 2015 & 2016 & Variación \\
\hline Fallecidos & 650 & 522 & 449 & 399 & 327 & 295 & 283 & 287 & 262 & 303 & $-53.38 \%$ \\
Heridos graves & 3608 & 3119 & 2362 & 1972 & 1864 & 1553 & 1615 & 1345 & 1281 & 1453 & $-59.73 \%$ \\
Heridos leves & 19462 & 17280 & 17850 & 16534 & 15503 & 15942 & 19042 & 20426 & 22616 & - & $+16.2 \%$ \\
\hline
\end{tabular}

Table 2: Evolución de fallecidos por accidente de tráfico en Andalucía (tasas por 100,000 habitantes). Análisis por grupos de edad. Periodo 2007 to 2013

\begin{tabular}{lccllllll}
\hline Edad & 2007 & 2008 & 2009 & 2010 & 2011 & 2012 & 2013 & Variación \\
\hline $0-14$ & 0.77 & 0.85 & 0.74 & 0.95 & 0.29 & 0.87 & 0.51 & $-34 \%$ \\
$15-24$ & 10.15 & 10.23 & 6.88 & 4.91 & 5.11 & 4.22 & 3.48 & $-66 \%$ \\
$25-34$ & 9.35 & 6.64 & 6.91 & 5.1 & 4.08 & 2.88 & 3.18 & $-66 \%$ \\
$35-44$ & 6.33 & 6.04 & 4.51 & 5.35 & 3.94 & 4.27 & 2.92 & $-54 \%$ \\
$45-54$ & 7.57 & 5.17 & 5.7 & 3.97 & 4.34 & 3.47 & 3.67 & $-52 \%$ \\
$55-64$ & 5.95 & 5.73 & 4.53 & 4.39 & 3.04 & 3.31 & 3.89 & $-35 \%$ \\
$>65$ & 3.5 & 4.89 & 4.12 & 4.4 & 4.08 & 3.61 & 4.32 & $+23 \%$ \\
\hline
\end{tabular}

a Educación, Ejecución (en adición a Disuasión), Ejecución de Ingeniería y Estímulos Económicos y multas. Las estrategias de educación para la prevención del trauma son efectivas de varias maneras y en varios niveles. Los programas de prevención deben ser dirigidos a los grupos de mayor riesgo identificados en las poblaciones. Los esfuerzos educativos son relativamente fáciles de ejecutar, incluso para el personal inexperto con los métodos de prevención de trauma. ${ }^{6}$

Existen tres categorías de prevención en trauma y todas ellas comprenden lo que se conoce como control de trauma: ${ }^{7}$

- Prevención primaria: Su objetivo es tratar de evitar que ocurra el incidente traumático.

- Prevención secundaria: Su cometido es intentar minimizar la severidad de las lesiones que ocurren como consecuencia del incidente, el cual no se consiguió prevenir de forma primaria.

- Prevención terciaria: Orienta sus esfuerzos en optimizar los resultados de las atenciones de las lesiones producidas después del incidente con independencia de su gravedad, con el objeto principal de evitar consecuencias funestas y minimizar las secuelas.

Aunque hasta ahora, se ha demostrado la eficacia de los programas de prevención en trauma, debemos insistir es este aspecto, así como en otros de vital importancia como la formación, la asistencia sanitaria, la investigación o la gestión, con el objetivo principal de minimizar en nuestra comunidad los incidentes, la gravedad de las lesiones secundarias a los accidentes no prevenidos, así como las secuelas secundarias.

\section{Referencias}

1. Comité on Trauma, American College of Surgeons: Resources for Optimal Care of the Injured Patient: 1993. Chicago, American College of Surgeons, 1993.

2. Olavarria Govantes L, Borrajo Martos C, et al. Plan Integral de Atención a la Accidentabilidad 2007-2012. Sevilla, Junta de Andalucía Consejería de Salud, 2007.

3. Ivers RQ. The role of epidemiology in improving the evidence base in injury prevention and trauma care. Injury 2012;43:395-396.

4. Instituto Nacional de Estadística. Cifras de población referidas al 01/01/2017. Cifra oficiales de población de los municipios españoles: revisión del padrón municipal. 2017.

5. SánchezJ.Marco conceptual de la promoción y educación para la salud. In: Marqués F, Sáez S, Guayta R, ed. Métodos y medios en promoción y educación para la salud. Barcelona: Editorial UOC, 2004; pp. 25-62.

6. The National Committee for Injury Prevention and Control. 1989. Injury Prevention: Meeting the Challenge. American Journal of Preventative Medicine.

7. Organización Mundial de la Salud. Informe mundial sobre prevención de los traumatismos causados por el Tránsito. Ginebra: Organización Mundial de la Salud; 2004.

8. Dirección General de Tráfico, Ministerio del Interior Gobierno de España. Las principales cifras de la siniestralidad vial. España 2016. En: http://www.dgt.es/Galerias/seguridad-vial/estadisticas-eindicadores/publicaciones/principales-cifras-siniestralidad/Lasprincipales-cifras-2016.pdf 


\section{InVited COMMENTARY}

"PROYECTO SOBRE RUEDAS"

Un "accidente" se considera un término que implica una circunstancia aleatoria que resulta en daño. Un accidente es un concepto en espera de suceder, un evento inesperado. La lesión es una enfermedad que tiene un huésped (paciente) y un vector de transmisión (MVC, armas de fuego, etc.). En 2007, en los Estados Unidos, las lesiones, incluidas todas las causas de lesiones no intencionales y relacionadas con la violencia combinadas, representaron el $51 \%$ de todas las muertes entre personas de 1 a 44 años de edad, es decir, más muertes que enfermedades no transmisibles y enfermedades infecciosas conjunto. Lo que hace resaltar la importancia de una organización de trauma preventivo.

Por ejemplo todos los centros de trauma, independientemente de los recursos, deben participar en la prevención de lesiones o, de lo contrario, se convierten en una deficiencia por parte del Comité de Verificación del Colegio Americano de Cirujanos (CD 18-1). Además, los niveles I y II deben demostrar evidencia de salario de apoyo y resultados para la prevención de lesiones. Por esto el rol de los Centros de Trauma es aumentar la conciencia pública sobre el concepto de prevención de lesiones. Los centros de trauma identifican a los pacientes que generalmente sobreviven, pero el $50 \%$ de todos los pacientes que mueren por lesiones nunca llegan a un hospital / centro de trauma. Un impacto importante en estas lesiones letales se puede lograr solo a través de la implementación de programas efectivos de prevención de traumas, aumentando la conciencia pública, a través de la educación.

El Proyecto 'Sobre ruedas', puesto en marcha y coordinado desde 2009 por la Secretaría General de Salud Pública y Consumo de la Consejería de Salud, se inscribe dentro del Plan Integral de Atención a la Accidentabilidad en Andalucía. Este Plan Integral destaca, además de los avances en el plano asistencial, la importancia primordial de las acciones de prevención y promoción que propicien la implicación de otros sectores, así como la participación de la ciudadanía a través de sus asociaciones.

Los tres objetivos principales del proyecto 'Sobre ruedas' son bien claros y especificos:

- Reducir el número de accidentes de tráfico y sus consecuencias en la población juvenil de la Comunidad Autónoma Andaluza.

- Sensibilizar desde el espacio socio-educativo a los jóvenes sobre la evitabilidad de los accidentes.

- Promover cambios de actitud ante conductas de riesgo así como contribuir a una movilidad segura, responsable y sostenible.

Si bien los individuos son responsables de tomar medidas para mantenerse seguros y protegerse a sí mismos y a sus familias de las lesiones, los expertos han descubierto que los esfuerzos como el "Proyecto sobre ruedas" desempeñan un papel importante para ayudar a las personas y las familias a mantenerse saludables y seguras. Las investigaciones han demostrado con pruebas sólidas que con diferentes estrategias se puede lograr una reducción de las lesiones comunes. La persistencia y la paciencia es la clave para los programas de prevención de lesiones en USA y en el resto del mundo.

Juan C Duchesne MD

Professor of Surgery

Tulane University

New Orleans, USA
"Project On Wheels"

An "accident" is considered a term that implies a random circumstance that results in harm. An accident is a concept waiting to happen, an unexpected event. Injury is a disease that has a host (patient) and a transmission vector (MVC, firearms, etc.). In 2007, in the United States, injuries, including all causes of unintentional injuries and violence-related injuries, accounted for $51 \%$ of all deaths among people 1 to 44 years of age, more deaths compared to noncommunicable diseases and infectious diseases combined. This highlights the importance of organized prevention of trauma. For example, all trauma centers, regardless of resources, must participate in the prevention of injuries. If not, it will be counted as a deficiency by the Verification Committee of the American College of Surgeons (CD 18-1). In addition, levels I and II must demonstrate evidence of supportive salary and document results for injury prevention. This is why the role of Trauma Centers is to increase public awareness of the concept of injury prevention. Fifty percent of all patients who die from injuries never reach a hospital/trauma center. A major impact on these lethal injuries, therefore, can be achieved only through the implementation of effective trauma prevention programs and increasing public awareness through education.

The "On Wheels" Project, launched and coordinated since 2009 by the General Secretariat of Public Health and Consumption of the Ministry of Health, is part of the Comprehensive Care Plan for Accident in Andalusia. This Integral Plan highlights, in addition to the advances in the health care plan, the paramount importance of prevention and promotion interventions that favor the involvement of other sectors, as well as the participation of citizens through their associations.

The three main objectives of the "On Wheels" project are very clear and specific

- Reduce the number of traffic accidents and their consequences in the youth population of the Autonomous Community of Andalusia.

- Sensitize young people about the avoidability of accidents from the socio-educational space.

- Promote changes in attitude toward risk behavior as well as contribute to a safe, responsible, and sustainable mobility.

While individuals are responsible for taking measures to stay safe and protect themselves and their families from injuries, experts have found that efforts such as the "Project on Wheels" play an important role in helping individuals and families to stay healthy and safe. Research has shown with solid evidence that with different strategies a reduction of common injuries can be achieved. Persistence and patience is the key to injury prevention programs in the United States and around the world.

Juan C Duchesne MD

Professor of Surgery

Tulane University

New Orleans, USA 